\title{
Potential of Application Focused Ion Beam in Forensic Science Area
}

\author{
Marek Kotrlý1,2 \\ 1. Institute of Criminalistics Prague (ICP), Czech Republic; \\ 2. Charles University in Prague, Faculty of Science, Praha, Czech Republic.
}

Electron microscopy techniques are widely used in forensic analysis, both for the initial screening and the final expert assessment. There is a wide variety of samples that we analyse at forensic laboratories and experts there may encounter virtually any material, either made by humans or created by nature, which is related to the case under investigation (from fragments of prehistoric vessels, through data and documents to high-tech semi-permeable semiconductors). Striving to extend the analytical tools, we have recently tested the possibility of using the focused ion beam. The respective devices have become conveniently small and the current dual SEM / FIB systems have become standard laboratory equipment. [1]

Probably the most widely used focused ion beam technology in this area is based on the use of Ga+ ions. With the help of this forensic technology, we can add and remove materials ("cuts" at the molecular level), perform secondary ion imaging and, potentially, execute ion microanalysis. In practice, it is about analysing the internal structure of micro and nano-particles, layers and composites (cross strokes in the graphic expertise, analyses of layers of functional glass, etc.), analysis of microdefects of alloys, 3D modelling of particles and aggregates, etc. The SEM/FIB technology has found its applications primarily in the following fields of forensic science:

Thermogenetic particles - Analysis of thermogenetic particles resulting from physical and chemical processes in which there is an immediate increase in temperature and pressure (post blast residues, gunshot residues, particles of airbags, etc.). In the forensic screening, we handle microscopic particles, imperceptible to the naked eye. Their detection, however, helps determine the offender's modus operandi. The thermogenetic particles have their own specific chemical composition, which depends on their origin. In many cases, it is impossible to clearly identify chemical components of the internal structure of particles solely from their surface and we often analyse important phases that crystallize in cavities of the particles. Morphology of the particle structure of the wall is also important as it is, in many cases, unique to the given particle. All this information is important for a clear classification of the given particle into the appropriate category. The focused ion beam allows for such detailed analysis of information, especially in cases where it is not entirely clear which category they belong to. Such information, however, can be crucial for the investigators. [2]

Nanocomposites and nanoparticles - In forensic practice, the most commonly encountered nanocomposite is found in colour variable pigments - security features (e.g. on banknotes), car body paint, consumer electronics, etc. and in functional layers of glass (thermo-insulated glass, automobile screens, etc.). We assume that the volume of these materials seen in forensic practice will further increase.

The analysis of nanocomposites in forensic practice strives to provide detailed information on structures to facilitate comparison with comparative samples presented. Since the direct analysis of the surface does not reveal enough information to facilitate qualified comparison of these materials (e.g. to confirm counterfeits), it is necessary to analyse directly the individual layers in the focused ion beam imaging. 
Holographic security features - Holograms, holographic seals and seals have been used increasingly to authorize prints, prove origin of products, and seal those parts of products in which it is necessary to reliably detect unauthorized access. The image itself is a result of refraction and interference of light on various structures of the holographic layer. It is possible to produce a hologram which will be a visually perfect reproduction of the original, but it will differ in its internal composition, or structure.

The analysis of holograms in forensic practice aims at the use of detailed structure-related and other information to facilitate comparison with the comparative samples presented and detection of counterfeits.

Deformations in alloys - Tool marks examination, detection and examination of defects, metallography and technical diagnostics - all these analytical tools strive to visualize deformations of materials, document and describe the changes and determine what may have caused such deformation (fatigue fractures, the effects of pressure and temperature, etc.). Ion imaging, unlike electrons, allows for good separation of grains in metal alloys and their micromorphology (as with an optical microscope), but with significantly higher magnification and resolution.

Analysis of superposition of toner / writing ink - Combination of written and printed documents is most often found in respect of wills, bills, contracts, etc. Those who forge such documents most often illicitly "edit" existing documents or "fabricate" new documents/papers. To determine whether the document/printed matter was first signed and other data were printed onto only subsequently or whether the signature was attached after printing, it is important to determine the superposition of strokes in case of "reprints" of previously signed documents. In these cases, superposition of toner particles and the writing ink may be one of the decisive factors.

The use of SEM / FIB technology poses new opportunities to forensic science. With this technology, we are now able to mine evidence from traces which had been previously very difficult to process, if not impossible. The technology has been used successfully in a number of real cases, e.g. in the analysis of post blast residua, analysis of functional layers of glass, counterfeit bills of exchange, etc., where conventional techniques failed to deliver a definite answer [3] [4].

\section{References:}

[1] Kotrlý M., Mareš B., Turková I., Beroun I.: Proc. SPIE 9823, (2016), p. 98230S-1 - 9.

[2] Kotrlý M., Turková I.: Proc. SPIE 9486, 2015, Vol. 9486, (2015), p. 948614-1 - 11.

[3] Kotrlý M.: Microsc. Microanal. 22 (Suppl 3), (2016), p. 2032.

[4] Acknowledgements - Microanalytical methods at ICP has been supported by the projects of the Ministry of the Interior of the Czech Republic: VG20102015065, VF20112015016, VI20152020004, VI20152020035, VI20172020050. 